\title{
Kultur(en) und Sprache(n) der Asylwirklichkeit - Herausforderungen empirischer Forschung im Kontext von Unsicherheit, Verrechtlichung, Interkulturalität und Mehrsprachigkeit
}

\author{
Andrea Fritsche
}

(C) Der/die Autor(en) 2016. Dieser Artikel ist eine Open-Access-Publikation.

Zusammenfassung Qualitative Forschung im Asylkontext wird häufig deshalb problematisiert, da einerseits das Interviewen hilfsbedürftiger, möglicherweise traumatisierter Personen besondere Zugänge erfordert oder andererseits, weil Verstehen durch sprachliche und kulturelle Fremdheit begrenzt wird. Der vorliegende Methodenbeitrag ändert die Perspektive und sieht die methodischen Herausforderungen der Datenerhebung nicht in den Charakteristika der Person sondern im gesellschaftlichen Umfeld, das ,,den Asylwerber“ erst schafft, bedingt. Auf Ebene der Auswertung wird ein methodisches Vorgehen diskutiert, das Mehrsprachigkeit und das Nebeneinander mehrere Kulturen nicht vorrangig als Hindernisse am Weg zum Verstehen, sondern als Chance begreift. Auf Grundlage konkreter Erfahrungen eines laufenden Forschungsprojekts zur österreichischen Asylwirklichkeit werden die methodisch relevanten Besonderheiten des Feldes dargestellt, gezeigt, wie etablierte methodische Ansätze für den Untersuchungskontext nutzbar gemacht werden können und welche konkreten Adaptionen und methodische Innovationen für Forschungen im Asylkontext nützlich sind. Zentral ist dabei zum einen der Blick auf die dem Feld eigene „Asylkultur“ und „Asylsprache“ und andererseits ein Verständnis der Interviewten nicht vorrangig als MigrantInnen sondern als RepräsentantInnen und ExpertInnen eben dieser Kultur und Sprache.

Schlüsselwörter Refugee Studies · Flüchtlinge als ExpertInnen · Qualitative Interviews $\cdot$ Kulturelle Positionierung $\cdot$ Asylkultur

Der Artikel basiert auf einem Vortrag im Rahmen des Workshops „Method(olog)ische Herausforderungen der Migrationsforschung“ am 18.4.2013 an der Karl-Franzens-Universität Graz.

A. Fritsche $(\square)$

Universität Wien, Wien, Österreich

E-Mail: andrea.fritsche@univie.ac.at 


\title{
Culture(s) and Language(s) of the asylum reality - Challenges of empirical research in the context of uncertainty, juridification, interculturality and multilingualism
}

\begin{abstract}
Qualitative research in the context of asylum is often made a problem, as on the one hand interviewing vulnerable, possibly traumatized individuals asks for particular methodological approaches and, as on the other hand, understanding seems to be limited due to linguistic and cultural foreignness. This methodological contribution changes the perspective and understands methodological challenges of data collection as not based on the characteristics of the individual but on that of the societal environment, which creates 'the asylum seeker' in the first place. A methodological approach, which perceives multilingualism and the coexistence of diverse cultures not primarily as an obstacle but as an opportunity for understanding is discussed on the level of data analysis. Based on concrete experiences of an ongoing research on the Austrian asylum reality, methodological particularities of the field are outlined; it is shown how established methodological approaches can be used in the given research context and what kind of adaptations and methodological innovation are useful for research in the context of asylum. It is imperative that on the one hand the focus is put on the ,,asylum culture“ and the ,asylum language“" distinct to the field; on the other hand the interviewees have to be understood not primarily as migrants but as representatives and experts of this particular culture and language.
\end{abstract}

Keywords Refugee Studies · Refugees and asylum seekers as experts - Qualitative interviews $\cdot$ Cultural positioning $\cdot$ Asylum culture

\section{Einleitung}

Wenn Flüchtlinge bzw. AsylwerberInnen zur Zielgruppe qualitativer Forschung werden, stellen sich v. a. zwei Fragen: Wie können hilfs- und schutzbedürftige Personen, die möglicherweise traumatisiert sind, befragt werden? Und: Wie ist Verstehen in einem kulturell und sprachlich fremden Kontext möglich? Ich möchte im vorliegenden Aufsatz versuchen, die Fragen, die im Rahmen empirischer Forschung mit AsylwerberInnen auftauchen, teilweise neu zu stellen: Einerseits betrachte ich die Herausforderungen der Datenerhebung weniger durch die Vergangenheit bzw. die Merkmale der Person (traumatisiert, vulnerabel, fremd etc.) als vielmehr durch die Charakteristika des gesellschaftlichen bzw. institutionellen Umfelds von AsylwerberInnen im Aufnahmeland bedingt. Andererseits sehe ich die (vermeintliche?) Fremdheit nicht nur als Ursache von lückenhaftem Verstehen, sondern auch als Chance der Forschung.

Grundlage der Auseinandersetzungen bildet ein laufendes Forschungsprojekt, dessen Erkenntnisinteresse darin liegt, zu verstehen, inwiefern Asyl als Recht und die Asylbeantragung als Einforderung von Rechten verstanden werden. Die österreichische Asylwirklichkeit wird dabei v. a. über Erzählungen von AsylwerberInnen erschlossen, intersubjektive Vorstellungen von Asyl sowie „des Flüchtlings“ und 
„des Asylwerbers“ werden ergründet; Prozessen, innerhalb derer diesbezügliche Bedeutungen verfestigt, herausgefordert und perpetuiert werden, wird nachgespürt.

Von September 2010 bis Juli 2012 wurden in diesem Zusammenhang mit 18 Asylwerberinnen und zwölf Asylwerbern aus 14 Ländern ${ }^{1}$ Gespräche geführt. Die Interviewten waren zwischen 18 und ca. 60 Jahre alt und wohnten in Wien, Niederösterreich, Kärnten und Vorarlberg. Der Zugang zu den Befragten kam einerseits mit Hilfe von NGOs der Grundversorgung, der Rechts- und Sozialberatung, der Bildungsförderung und der Nachbarschaftshilfe sowie einer Psychotherapieeinrichtung zustande. Andererseits waren ehemalige AsylwerberInnen wichtige Gatekeeper zu InterviewpartnerInnen. Im Sinne einer ,impliziten Triangulation“ (Flick 2008, S. 315) wurden die Interviews durch mehrwöchige Beobachtungen in einer Grundversorgungs- und einer Rechtsberatungseinrichtung bzw. bei Begleitungen im Asylverfahren sowie durch nicht-reaktiv entstandene Dokumente - v. a. Bescheidauszüge, Fotos und (literarische) Texte von AsylwerberInnen - ergänzt.

\section{Struktur des Artikels}

Der Artikel ist zweigeteilt: Nach der Darstellung der Besonderheiten des Forschungsfeldes beschäftigt sich der erste Teil mit der Datenerhebung, d. h. konkret den Möglichkeiten qualitativer Interviews im Kontext. Im zweiten Teil wird der Fokus auf die Auswertung gelegt und der Frage nachgegangen, welche Sprache(n) und Kultur(en) im Zentrum der Forschung stehen, sowie ob und wie auch im Kontext von Interkulturalität und Mehrsprachigkeit latente Bedeutungen erschlossen werden können.

Sowohl in Bezug auf die Datenerhebung als auch die Auswertung werden Herausforderungen der empirischen Forschung reflektiert; es wird dargestellt, wie etablierte methodische Ansätze für den vorliegenden Forschungsbereich nutzbar gemacht und neue bzw. adaptierte methodische Vorgehensweisen in ihrer Relevanz für den Forschungskontext diskutiert und in forschungspraktische Schritte übersetzt werden können.

\section{Kontext \& Besonderheiten des Feldes}

Je nach Perspektive sind AsylwerberInnen Flüchtlinge im materiellen Sinn², MigrantInnen, die angesichts der Abschottungspolitik einer „Festung Europa“ gelernt haben, das Asylrecht bzw. das eingeschränkte Angebot an MigrantInnenkategorien (vgl. Müller 2010, S. 39 f.) als eine der wenigen Möglichkeiten der Legalisierung ihres Daseins in Europa zu nutzen (vgl. Cuttitta 2010, S. 34) oder aber sie sind - oft

\footnotetext{
1 Georgien, Türkei (Kurde), DR Kongo, Republik Kongo, Nigeria, Gambia, Algerien, Mongolei, Russische Föderation, Ukraine, Iran, Afghanistan, Somalia, staatenlos (Geburtsort: Armenien).

2 Materiellrechtlich sind auch Asylwerber Flüchtlinge, denn ,,[n]icht auf Grund der staatlichen Anerkennung werden Verfolgte Flüchtlinge, sondern die Anerkennung erfolgt, weil sie Flüchtlinge sind“" (Schumacher et al. 2012, S. 13).
} 
aus der Perspektive des an Sicherheitsparadigmen orientierten nationalstaatlichen Migrationsmanagements - potenziell illegitime, irreguläre und ,illegale“ MigrantInnen, deren Daseinsberechtigung im besten Fall noch zu prüfen ist. Asylwerber ${ }^{3}$ sind somit ein Typus und eine Kategorie im Ordnungsschema um nationale Zugehörigkeiten (vgl. Müller 2010, S. 48; Benhabib 2006). Das Bundesministerium für Inneres ordnete 2012 12.846 Männer und 4567 Frauen unterschiedlichen Alters aus mindestens 98 Ländern ${ }^{4}$ (BM.I 2012) dieser Kategorie zu; Menschen mit unterschiedlichen Flucht- bzw. Migrationsgründen, Biographien, Bildungsabschlüssen, Berufen, Weltanschauungen oder Religionszugehörigkeiten. Die heterogene Gruppe der AsylwerberInnen vereint faktisch nur, dass sie sich außerhalb ihres Herkunftsstaates befinden und einen Antrag auf internationalen Schutz gestellt haben. Das daraus resultierende rechtliche Label Asylwerber geht nicht nur mit bestimmten Rechten und v. a. Pflichten einher, sondern bedingt Strukturen, die von Exklusion und Unsicherheit sowie von Bürokratisierung und Verrechtlichung geprägt sind.

\section{Verrechtlichung \& Bürokratisierung}

Einen Asylantrag zu stellen, bedeutet, sich einem verwaltungsrechtlichen Verfahren zu unterwerfen, das vorrangig dazu dient, die ,deserving refugees“ (Sales 2002) zu selektieren. Als AsylbewerberInnen ${ }^{5}$ im wahrsten Sinne des Wortes muss die Person weitreichende Kompetenzen zeigen, um im Wettbewerb um den Titel des „wahren Flüchtlings“ zu bestehen. Im Asylverfahren müssen nicht nur die individuellen Fluchtgründe glaubhaft dargestellt werden, sondern dies hat in Übereinstimmung mit spezifischen Wissens-, Wahrheits- und Machtregimen zu erfolgen (vgl. Conlon 2013, S. 198). Die Glaubhaftmachung muss darüber hinaus aus der Kultur des Aufnahmelandes heraus verständlich sein, und die eigentliche Identität der Asylwerberin/des Asylwerbers darf dem Selbstverständnis des Nationalstaates nicht gegenläufig sein (vgl. Noll 2006, S. 498 f.).

Als bürokratischer Rechtsakt ist das Asylverfahren von laufenden Einvernahmen, gegebenenfalls medizinischen oder linguistischen Untersuchungen, Ladungen, Protokollen, Bescheiden, sowie blauen Briefen und gelben Zetteln ${ }^{6}$ geprägt. Die Einvernahmen werden von den Asylsuchenden häufig als „Interviews“7 bezeichnet, deren Ausformung nur wenig mit den Kriterien gemein zu haben scheint, die der UNHCR für eine ordnungsgemäße Prüfung einfordert: Statt einem offenen und beruhigenden Umfeld, sich neutral, mitfühlend und objektiv verhaltenden InterviewerInnen

\footnotetext{
3 Die ausschließlich männliche Form wird in Kursiv dann verwendet, wenn der rechtliche Status im Zentrum steht, denn auch das Asylgesetz kennt keine weibliche Form.

${ }^{4}$ In der Statistik scheinen zusätzlich Staatenlose und Personen, deren Staatsangehörigkeit ,ungeklärt“ ist, auf.

5 Das deutsche Recht verwendet die Terminologie „Asylbewerber“.

${ }^{6}$ Bescheide im Asylverfahren werden meist als RSa-Briefe, d. h. zu eigenen Handen, zugestellt; diese sind, aufgrund ihrer Farbe, auch als ,blaue Briefe“ bekannt; die Benachrichtigung über derartige Briefe wird auf gelben Zetteln hinterlegt.

7 Der Begriff „Interview“ wird dann in Anführungszeichen gesetzt, wenn damit die Einvernahme gemeint ist und nicht das Interview als methodisches Instrument.
} 
(vgl. UNHCR 2013, S. 105) werden die Einvernahmen als „Wissensprüfung, auf die sich vorbereiten muß, wer bestehen will“ (Scheffer 2003, S. 456) erlebt. Um nicht als „AsylbetrügerIn“ bzw. „Wirtschaftsflüchtling“ klassifiziert zu werden, ist eine glaubwürdige Darstellung als ,echter Flüchtling“ notwendig, womit v. a. eine konstante Repräsentation als Opfer einhergeht.

In Konfrontation mit dem Asylsystem werden bestimmte Erzähl- und Darstellungsformen gelernt, die erfolgreicher als andere erscheinen: In der Einvernahme sind meist eine bestimmte Chronologie, exakte Zeitangaben und ein detailliertes Erzählen, das ohne Widersprüche reproduzierbar ist, gefordert; bestimmte Kompetenzen sind notwendig, um die Testmethoden erfolgreich zu meistern (vgl. ebd.). Individuellen oder kulturell bedingten Erzählformen wird kaum Platz gelassen, narrative Ungleichheiten werden reproduziert (Bloemmaert 2001). Asylanhörungen, als „umfassender Zugriff auf die Biographie der Betroffenen“ (Thielen 2009, Abs. 7) sind zusätzlich von verfestigten Rollenvorstellungen gekennzeichnet: Die Referentin/der Referent fragt aus einer institutionellen und professionellen Position, die AsylwerberInnen erzählen, ohne Rückzugsraum auf eine klar abgesteckte Rolle, als Privatpersonen intime Erfahrungen. ${ }^{8}$ Ein ähnliches Rollenmissverhältnis zeigt sich auch in anderen Erzählkontexten: bei Gesprächen mit Rechts- und SozialberaterInnen bzw. AnwältInnen oder in Zusammenhang mit Therapien, in der die Therapeutin dem Patienten, der seine privatesten Bereiche offenlegt, professionell und distanziert zuhört, ohne sich selbst durch das Einbringen biographischer Informationen verletzbar zu machen.

\section{Unsicherheit \& Exklusion}

Exklusion und Unsicherheit prägen sowohl den Alltag von AsylwerberInnen als auch den rechtlichen Kontext: Das Aufenthaltsrecht gilt lediglich temporär; weder ist bekannt, wie lange das Verfahren dauert, noch in welchen Status - vom anerkannten Flüchtling im Sinne der Genfer Flüchtlingskonvention bis zum Nicht-Status der/des rechtlosen Illegalisierten - das Asylverfahren mündet. Von den Asylsuchenden selbst wird das Verfahren oft als unzugängliche Black Box und das Asylsystem als fremde Rechtskultur erlebt, in der aufgrund der deutschen Verfahrenssprache Kommunikation nur mit Hilfe von (Laien-)DolmetscherInnen möglich ist. Das Asylsystem ist so nur teilweise und über Umwege zugänglich, Nicht-Wissen führt zu Ausschluss und eingeschränkter Handlungskompetenz, Gefühle der Unsicherheit sind vorherrschend.

Auf Alltagsebene führt das rechtliche Label und die Umsetzung rechtlicher Normen in die Praxis eines Grundversorgungssystems, in dem kulturelle, soziale und wirtschaftliche Rechte eingeschränkt, Unterbringung und Versorgung als Wohlfahrtssystem organisiert sind. AsylwerberInnen leben meist in organisierten Quartieren, oft geografisch abgeschieden und von der Mehrheitsgesellschaft ausgeschlos-

\footnotetext{
8 Ähnliches gilt auch für den Forschungskontext: „Im Interview sprechen nicht einfach nur Menschen zu Menschen, sondern geben auf einigermaßen gezielte Fragen von Menschen einer bestimmten Art Menschenwissenschaftler - Antwort.“ (Luckmann zitiert in Honer 1993, S. 71).
} 
sen. Diese Bedingungen werden als „organisierte Desintegration“ (Täubig 2009; Rosenberger und König 2010, S. 289 f.) mit ,,inhärente[n] Kontroll- und Disziplinierungsmechanismen“ (ebd., S. 273) beschrieben, in der den Prinzipien „Ruhe, Ordnung und Sicherheit" (ebd., S. 284) zu folgen ist. Diese Rahmenbedingungen bringen praktische Probleme mit sich und können weitreichende Auswirkungen auf die Person haben:

[Claiming asylum] is the worst thing a man should think about. [...] I'll advise anybody NEVER to try it. [...] Their lives die [...] [T]here are some people who send them to an asylum house OUTSIDE the main city [...] the bus leaves maybe twice a day or even once in a week. And they just be giving him food. What you are doing to him? You are KILLING him! (Transkript Amaru, 25.1.2011)

Die Unsicherheit wird durch öffentliche Diskurse, die sich v. a. aus dem Kriminalisierungs- und Missbrauchsframe nähren (vgl. asylkoordination 2009, S. 18 ff.; Bauböck 2004; Bigo 2010), verstärkt. AsylwerberInnen werden vermehrt als Last für den Staat und das Sozialsystem sowie als Gefahr für das Aufnahmeland konstruiert. Auf der Suche nach Schutz finden sich AsylwerberInnen oft in einem gesellschaftlichen und politischen Kontext wieder, der von Fremdenfeindlichkeit und Hoffnungslosigkeit geprägt ist (vgl. ECRE 2013, S. 23 f.).

Eine Folge dieser Bedingungen ist, dass das Streben nach Inklusion und „Integration" zur sozialen und rechtlichen Erfordernis wird: Einerseits ist der Versuch, Anschluss zu finden, eine individuelle und soziale Notwendigkeit, um weiterleben und die eigene Würde aufrechterhalten zu können. Andererseits ist „Integration“ aus rechtlicher Perspektive oft unabdingbar: Im Rahmen des Verfahrens wird nicht nur geprüft, ob sich die Person für einen Schutzstatus qualifiziert, sondern auch, ob eine etwaige Ausweisung zu einer Verletzung von Artikel 8 der Europäischen Menschenrechtskonvention - dem Recht auf Privat- und Familienleben - führt. ${ }^{9}$ Überprüft werden dabei v. a. die „Integrationsleistungen“: Nur wer ein Dasein, das auf intensive Teilnahme an der österreichischen Gesellschaft schließen lässt, glaubhaft machen kann, wer also trotz Bedingungen der Exklusion und Unsicherheit als ,integriert“ erscheint, hat Chancen auf ein sogenanntes „Bleiberecht“ und somit eine zusätzliche Möglichkeit, den Rechtsstatus Asylwerber zu verlassen.

\footnotetext{
9 Abseits der Zuerkennung des Status des Asylberechtigten wird die Zuerkennung von subsidiärem Schutz - bei Gefahr einer Verletzung von Art. 2 EMRK, Art. 3 EMRK oder der Protokolle Nr. 6 oder Nr. 14 zur Konvention - geprüft (vgl. §3 bzw. §8 AsylG 2005 idgF). Gemäß 9 Abs. 2 BFA-VG idgF wird Art. 8 EMRK anhand von neun Punkten geprüft, dabei wird mit Z 4 - dem ,Grad der Integration“ - direkt auf die notwendigen „Integrationsleistungen“ Bezug genommen.
} 


\section{Datenerhebung in der Asylwirklichkeit}

\subsection{Methodische Herausforderungen im Feld}

Qualitative Forschung im Allgemeinen und Interviews im Speziellen in einem Umfeld, das durch Verrechtlichung und Bürokratisierung, Unsicherheit und Exklusion geprägt ist, sind somit entsprechend konnotiert und belastet: Restriktive Bedingungen und Sanktionsandrohung beim Ausbrechen aus dem Exklusionssystem, der unsichere Aufenthaltsstatus und laufende Kontrollen führen zu Angst und perpetuieren Unsicherheiten. Dies spiegelt sich bereits in den Möglichkeiten des Feldzugangs, wenn entweder Unterkünfte kaum zugänglich sind oder Polizeikontrollen zum sozialen Rückzug zwingen, wider:

Eine der Frauen sagt, ja, sie möchte das [Interview] machen, ganz bald, ich soll zu ihr in die Pension kommen [...] dann könne ich nicht nur hören, sondern auch sehen. [...] Daraufhin weist mich [...] die [NGO-Mitarbeiterin] nochmal darauf hin, dass ich nicht in die Pensionen darf, weil dies für Außenstehende nicht möglich sei. (Forschungsnotiz Kärnten, 20.6.2011)

Ich frage nach, was passiere, wenn man in die Pensionen gehe, [d]er [NGOVertreter] meint, dass es strafbar sei, und wenn's schlecht ausgehe, als Hausfriedensbruch interpretiert werde. Ein Besuch könne unter Umständen den AsylwerberInnen selbst schaden, weil sie damit gegen die Hausordnung verstoßen, die festlegt, dass sie keinen Besuch empfangen dürfen. (Forschungsnotiz Kärnten, 21.6.2011)

M. [Leiterin der Unterkunft] meint [...] in der Früh [sei] die Polizei um sechs Uhr da gewesen und jetzt hätten die Leute Angst, d. h. auch klopfen sei ungut, weil sie nicht mehr raus wollen. (Forschungsnotiz Niederösterreich, 11.8.2011)

Eine Atmosphäre der Unsicherheit und Exklusion stellt nicht nur den Feldzugang vor entsprechende Herausforderungen, sondern macht das Herstellen von Vertrauen zentral - und oft entsprechend schwierig. So ist im Kontakt mit Lidinga ${ }^{10}$ ein Changieren zwischen sprechen wollen und der Angst vor Konsequenzen spürbar, die Unsicherheit greifbar:

L. [ruft mich] am Abend an, ich meine, ich sei leider schon in Wien, würde aber Anfang August nochmal nach Kärnten kommen, ob [...] ich ihn kontaktieren dürfe? Er meint ja [...] ich könne es probieren. Aber auf keinen Fall dürfe ich ihn filmen, es dürfe keine Bilder geben. [...] [Beim Interview] gehen wir in sein Zimmer ${ }^{11}$, das ist abgesperrt, er sperrt es auch ab, als wir drinnen sind. Am Schluss bittet er mich noch inständig, dass ich ja nicht den Namen und seine Identität preisgebe. (Forschungsnotizen Kärnten, 21.6. und 2.8.2011)

\footnotetext{
10 Immer wenn im Text lediglich Vornamen verwendet werden, handelt es sich um Pseudonyme von InterviewpartnerInnen.

11 L. umgeht damit die Anordnung des praktischen Besuchsverbots in der Unterkunft.
} 
Zusätzlich werden in einem derartigen Kontext, in dem das Verstehen eines fremden Rechtssystems die Zukunftschancen wesentlich bestimmt, Außenstehenden - so auch ForscherInnen - oft vielschichtige Erwartungshaltungen entgegengebracht. Im gegenständlichen Projekt besonders zentral waren dabei:

- Erwartungen in Zusammenhang mit dem Typus der (ehrenamtlich) Helfenden, die über Kreativ-, Sport- und Nachhilfeprojekte oder auch Sozial- bzw. Rechtsberatung bekannt sind, und die zu alltagspraktischen Erleichterungen beitragen könnten.

- Hoffnungen, ich könne als Person, die die Begrifflichkeiten des Asylsystems (wie Beschwerde, Bundesasylamt, weiße Karte etc.) zu verstehen scheint, als Rechtsberaterin fungieren und die Black Box Asylsystem zugänglich(er) machen.

- Möglichkeiten, aus der Forschungsbeziehung bzw. der Bekanntschaft mit einer Angehörigen der Mehrheitsgesellschaft einen möglichen Integrationsbeweis zu generieren.

- Situationen, in denen ich mich als Zuhörerin an der Grenze zur Therapeutin oder Sozialarbeiterin bewegte ${ }^{12}$ (vgl. Thielen 2009, Abs. 16 ff.).

Rollengrenzen sind somit einerseits - wo möglich - zu antizipieren und ständig neu zu verhandeln, und andererseits ist das gesamte Forschungsgespräch vom Verständnis des „Interview[s] als Anhörung“ (Thielen 2009, Absatz 11-13) abzugrenzen. Die Interviewsituation soll dabei möglichst wenige Erinnerungen an behördliche Einvernahmen aufkommen lassen und es den Interviewten gleichzeitig weitgehend ermöglichen, aus den im Asylverfahren vorherrschenden Erzähllogiken auszubrechen oder diese auf Metaebene zu reflektieren.

Angesichts der Besonderheiten des Feldes sind qualitative Interviews so auszugestalten, dass

- einerseits Nähe, Vertrauen und Empathie - als Gegenpol zu den Bedingungen der Exklusion und Unsicherheit - möglich sind und andererseits die notwendige Rollenabgrenzung über professionelle Distanz erleichtert wird;

- trotz der aus dem Kontext resultierenden Erwartungen und der Konnotation von Papier, Unterschrift und Bürokratie ein ,informed consent“ hergestellt werden kann;

- Parallelen zur Einvernahme im rechtlichen Kontext vermieden und Charakteristika der Verrechtlichung und Bürokratisierung nicht reproduziert werden;

- freie Erzählungen möglich sind, ohne narrative Ungleichheiten zu zementieren.

Welche Aspekte des qualitativen Methodenrepertoires für den Umgang mit diesen Herausforderungen nutzbar gemacht werden können, wie diese für den vorliegenden Kontext zu adaptieren sind und welche neuen Zugänge sich hier als nützlich erweisen, wird nachfolgend dargelegt.

\footnotetext{
12 Anzumerken ist, dass versucht wurde, Interviews mit Personen, die bekanntermaßen traumatisiert waren, zu vermeiden, da mir als Interviewerin die Kompetenz fehlte, hier entsprechend zu reagieren.
} 


\section{Interviews mit AsylwerberInnen - methodische Referenzen und kontextspezifische Adaptierungen}

Die Entscheidung für offene, narrativ orientierte Interviews ist sowohl der Forschungsfrage geschuldet als auch eine methodisch angemessene Reaktion auf die dargestellten Herausforderungen. Gerade die Narrationsorientierung qualitativer Interviews wird in einem System struktureller Exklusion zu einer Mitteilungs- und Anschlussmöglichkeit: Ein Interview zu geben bedeutet, mit einer im mehrfachen Sinne ,außenstehenden“ Person zu sprechen und auch gehört zu werden.

Das Potenzial offener Interviews entfaltet sich v. a. dann, wenn die Merkmale behördlicher „Interviews“ bewusst umgedreht werden: Statt durch die Verwendung von DolmetscherInnen die kommunikative Dreieckssituation des Asylverfahrens zu duplizieren, ermöglichen Interviews in einer Lingua Franca - trotz damit einhergehender Begrenzungen - direkte Kommunikation und unterstreichen die aktive SprecherInnenrolle der AsylwerberInnen. Während das Verfahren lediglich an der Asylwerber-Identität der Person interessiert ist, schaffen offene Interviews Raum, um andere Identitäten als die des Asylwerbers repräsentieren und aktualisieren zu können (vgl. Fritsche 2012, S. 370 f.): So präsentierte sich z. B. Serhildan während des Interviews als Student und politischer Aktivist, Veronika unterstrich ihren Akademikerinnenstatus und ihre Berufsidentität als Journalistin. Die Hervorhebung der eigenen Rechtschaffenheit oder Legitimität der individuellen Fluchtgründe ermöglicht eine Distanzierung von Kriminalisierungs- und Missbrauchsdiskursen. Auch wenn die im Verfahren manifesten Machtasymmetrien u. a. aufgrund der Tatsache, dass die Forscherin der Mehrheitsgesellschaft angehört, keineswegs nivelliert werden können, bietet das offene Interview Raum, Umdeutungen oder Nutzbarmachungen der Forschungssituation durch die RespondentInnen zuzulassen und so Narrationen zu begünstigen.

Als im Forschungskontext besonders gewinnbringend erweisen sich Zugänge der lebensweltlichen Ethnographie (Hitzler und Honer 1986; Honer 1993, 2008) und dabei v. a. das in diesem Rahmen entwickelte „dreiphasige Intensivinterview“ (Honer 1993, S. 70 ff.). Dieses stellt insbesondere dann ein kompensatorisches Erhebungsinstrument dar,

wenn das Forschungsideal der Mitgliedschaft am Feldgeschehen Beschränkungen unterworfen bzw. (warum auch immer) verunmöglicht ist. Wenn es nicht gelingt, durch eigene unmittelbare Erfahrung intime Feldkenntnis zu erlangen [...] dann bietet diese Interviewform - trotz des unvermeidlichen Anschauungsund Erfahrungsverlustes - eine besonders gute Chance [...] durch die je subjektive Perspektive der Befragten, Erfahrungen mit und Bedeutungen von bzw. in einem thematischen Ausschnitt ihrer Lebenswelt zu erfassen (ebd., S. 73 f.).

Die angesprochene Beschränkung der Mitgliedschaft gilt gerade für Erfahrungen des Asylwerber-Seins, die nur sehr begrenzt nachgelebt werden können. Ausschluss und Abschottung begrenzen den Zugang, das Klima der Unsicherheit beeinträchtigt die Offenheit bzw. birgt tatsächliche Risiken und Verrechtlichung und Bürokratisierung führen zu uneinsichtigen Prozessen. 
Das dreiphasige Interview beginnt mit einem „quasi-normalen Gespräch“ als erster Phase, wobei es hier darum geht, „,[m]öglichst normal miteinander [zu] reden“ (ebd., S. 74), d.h. in dialogischer Form, die explizit Bemerkungen, Zustimmung, gelegentlich sogar Widerspruch zulässt und in deren Rahmen sich die Forscherin einbringen kann. Auf eine nicht-direktive Gesprächsführung ist dabei weitgehend zu verzichten. Dieser Zugang kann im Fall der Forschung mit AsylwerberInnen genutzt werden, um Vertrauen herzustellen, gemeinsam den thematischen Rahmen des Gesprächs zu erarbeiten, Spielregeln auszuhandeln ${ }^{13}$, einen ,,informed consent ${ }^{\text {“14 }}$ herzustellen und Erwartungen zu klären. Über den dialogischen Einstieg können auch die Gesprächsform ausgehandelt und narrative Ungleichheiten gemindert werden. Nach einer zweiten, narrativen Phase, die v. a. subjektive, biographisch gewachsene Interpretationen der (Asyl-)Wirklichkeit erschließen will, folgt eine homogenisierende Phase, die es ermöglicht, Lücken zu schließen bzw. ausgewählte Aspekte strukturiert nachzufragen (vgl. ebd., S. 70 ff.; Hitzler und Honer 1986, S. 12 ff.).

\section{Mit methodischen Adaptierungen und Ergänzungen ermöglicht dieser Zugang auf mehreren Ebenen einen Umgang mit den dargestellten Herausforderungen}

\subsection{Von georgischer Gastfreundschaft zum Termingespräch}

Um Vertrauen herzustellen, im Asylsystem gelernte Erzähllogiken zu durchbrechen und mit der mehrfach konnotierten Situation „Interview“ umzugehen, ist Flexibilität bzw. die pragmatisch modifizierte Anwendung der drei Interviewphasen - sowohl deren Dauer, deren konkrete Ausformung als auch deren Stellenwert betreffend notwendig (vgl. Honer 1993, S. 84): So kann sich in manchen Fällen die erste Phase über mehrere Treffen ziehen, sodass von einem ,Hanging-Out“, d. h. länger andauernden ,,informal, interpersonal and 'everyday' types of encounter" (Rodgers 2004, S. 48), gesprochen werden kann. In anderen Fällen wird die zweite Phase praktisch übersprungen und die homogenisierende Phase aus dem quasi-normalen Gesprächs gespeist. Wieder andere Gespräche bedienen sich des quasi-normalen Gesprächs für ein kurzes Kennenlernen, um dann gleich in eine ausführliche Narration überzugehen.

Durch ein weitgehendes Überlassen der Erzählform und Steuerung der Interaktion an die InterviewpartnerInnen wird diesen Macht zugestanden und die Abgrenzung zur behördlichen Einvernahme erleichtert. In der Praxis kam es so zu einer großen Spannbreite: Thea wollte z. B. nur im Setting einer ,georgischen Gastfreundschaft““ sprechen, und jedes unserer Gespräche fand im familiären Setting am Küchentisch, begleitet von Essen, Tee oder Tarchuna ${ }^{15}$ statt und dauerte mehrere Stunden. Marika

\footnotetext{
13 Laut Bourdieu ist es gerade der fehlende Aushandlungsprozess in Bezug auf die Spielregeln des Interviews, der die Asymmetrie zwischen InterviewerIn und Interviewtem/r verstärkt und in Folge auch das Ausmaß symbolischer Gewalt erhöht (Bourdieu 2010 [1993], S. 395).

14 Vgl. Abschnitt ,Iterative Einwilligung“.

15 In Georgien verbreitetes, nach Estragon schmeckendes grünes Soda-Getränk.
} 
hingegen bevorzugte eine klare zeitliche Begrenzung auf ein bis zwei Stunden und einen neutralen Rahmen für das Gespräch.

\subsection{Sich im Gespräch positionieren}

Geprägt wird das Interview auch durch eine oft vorherrschende diffuse Angst, dass alles, was gesagt wird, potenziell negative Auswirkungen auf den Verfahrensausgang haben könnte. Daher ist es, über die Phase des quasi-normalen Gesprächs hinausgehend, wesentlich, sich als Person und nicht nur als Forscherin im Interview einzubringen, neutrale Positionen gegebenenfalls aufzugeben und - wo nötig - (politische) Positionierungen offenzulegen. So forderte z. B. Belisha mehrfach eine explizite Positionierung meinerseits, dass ich weder der FPÖ nahe noch auf Seite der Asylbehörden stehe. Zurückhaltung begünstigt hier nicht unbedingt Narrationen, sondern kann - ähnlich wie Fröhlich für Forschung im russischsprachigen Raum konstatiert - „Misstrauen der Interviewpartner/innen, die nicht wissen, woran sie sind, [provozieren]“ (Fröhlich 2012, S. 193).

\subsection{Iterative Einwilligung}

Durch bewusste Positionierungen, einen flexiblen Umgang mit methodischen Prämissen und die Abgabe von Steuerungsmacht werden zwar die Abgrenzung zur Einvernahme und die Herstellung von Vertrauen erleichtert, nicht aber unbedingt ein gemeinsames Verständnis der Gesprächssituation als Interview im Forschungskontext etabliert. Auch wenn der Einstieg über das quasi-normale Gespräch das Abstecken des Gesprächsrahmens im Dialog erleichtert, kann nicht angenommen werden, ,that research participants necessarily share a common understanding of the purpose of research, the role of the academic and the academy, notions of consent, forms of engagement (and recruitment), or communication and perceptions of risks“ (Pittaway et al. 2010, S. 242); folglich ist die laufende Herstellung eines ,informed consents“ wesentlich.

Dass dies gerade im Kontext der Flüchtlingsforschung entsprechend herausfordernd ist, wurde mehrfach thematisiert (vgl. Ellis et al. 2007, S. 467 ff.; Block et al. 2012, S. 72 f.; Mackenzie et al. 2007, S. 306 f.): Zu den Herausforderungen gehören z. B. Probleme mit schriftlichen Einwilligungen aufgrund von möglichem (funktionalem) Analphabetismus, die negative Konnotation von Schriftstücken und/ oder Unterschriften oder die Annahme, dass eine am Konzept des autonomen Individuums orientierte Einverständniserklärung kulturell eher kollektiv ausgerichteten Orientierungen zuwiderlaufen könnte. Um dennoch dem , informed consent “ als grundlegendem ethischem Forschungsprinzip treu zu bleiben, erweisen sich iterative Modelle der Einwilligung und ein ständiger Verhandlungsprozess in Hinblick auf ein gemeinsames Verständnis des Forschungsprozesses als sinnvoll (vgl. ebd., S. 306 ff.). Abseits einer wiederholten, explizit mündlichen Abgrenzung des Interviews von anderen Gesprächssettings, ist die Betonung, dass ein Rückzug jederzeit möglich ist, zentral. Gerade bei Unsicherheiten erweist sich die Vereinbarung, dass sich die Person, wenn sie noch einmal interviewt werden will, bei der Forscherin melden soll (und nicht umgekehrt), als hilfreich. 
Eine besondere Rolle kommt schriftlichen Kurzinformationen über das Projekt und die Rolle des/der RespondentIn zu. Auch wenn in Folge der Erfahrungen im Asylkontext Papier einerseits negativ besetzt ist, unterstreicht es andererseits die Wichtigkeit einer Sache, denn v. a. im Verfahren gilt nur das, was schwarz auf weiß geschrieben steht (Fritsche 2012, S. 381 f., 2013, S. 161 f.). Auch die Aushändigung einer Visitenkarte kann so zusätzliche Information über die Gesprächssituation liefern und in diesem Fall die Zugehörigkeit der Forscherin zum Universitätssystem, und nicht zum Asylsystem, unterstreichen. Zusätzlich erweisen sich schriftliche Informationen auch insofern als sinnvoll, als dass potenzielle RespondentInnen ,Entscheidungszeit“ gewinnen, um einer Teilnahme zuzustimmen oder diese abzulehnen.

\section{4 ,Gesunde“ Distanz durch Materialisierung der Forschungssituation}

Eine weitere Maßnahme, die den Charakter der Gesprächssituation als Interview im Forschungskontext erinnert, ist deren digitale Aufnahme. Entgegen mehrfacher Empfehlung, die Gespräche aufgrund der Vulnerabilität der Personen nicht digital aufzuzeichnen, ist in der Praxis häufig gerade die Verwendung eines Aufnahmegeräts hilfreich: Durch die so stattfindende Materialisierung der Situationsdefinition kann die Forschungsebene der Gesprächsbeziehung und somit auch die Abgrenzung zur Therapiesituation immer wieder erinnert werden. Eine so stattfindende Herstellung von Distanz unterstützt die Rollenabgrenzung und -klärung.

\subsection{Geben und Nehmen}

Als letzter Aspekt und als Gegenpol zu Bedingungen der Exklusion und Unsicherheit erweist sich ein Zugang im Sinne einer ,reciprocal research“ (Pittaway et al. 2010, S. 238) als nützlich. Reciprocal Research geht dabei über eine Solidarität mit den „Beforschten“ und ein „Do-No-Harm-Credo“ hinaus und will ,direct, tangible benefits to those who participate“ (ebd., S. 234) zurückgeben. Im vorliegenden Forschungskontext geht es dabei weniger um ein aktives Anbieten einer Gegenleistung (da dies Bedeutungen von Abhängigkeit und Almosen verstärken kann) als um ein Raum-Schaffen für das Einfordern derselben. So wurde z. B. Geld für Fahrtkosten mehrfach verweigert oder ich wurde bei Kaffeehaus-Gesprächen daran gehindert, die Rechnung zu begleichen. Stattdessen nahmen die Interviewten häufig eine aktive Rolle ein: $\mathrm{Zu}$ einem für sie passenden Zeitpunkt fragten sie nach der aus ihrer Sicht passenden Gegenleistung. So bat mich z. B. Mohamed, seine psychologischen Befunde ins Englische zu übersetzen, Lidinga beauftragte mich, seinen aktuellen Verfahrensstand zu eruieren, und Levi meldete sich ein paar Wochen nach unserem Interview per E-Mail mit der freundlichen „Aufforderung“, ihm zwei Texte zu übersetzen:

Bonsoir Andrea!

Je $\mathrm{t}$ ai envoye 2 textes et tu $\mathrm{m}$ excuse qu' ils soient longs mais $\mathrm{j}$ en aurai besoin jusq' au moins 15 septembre. Merci d avance pour ton service. (E-Mail, 17.8.2011) 
Und gerade in Hinblick auf die oben dargelegte amtliche Notwendigkeit der „Integration“ wurde ich mehrfach gebeten, die Forschungsbeziehung schriftlich zu bestätigen, um die Integration zu stützen. Derartige Gegenleistungen tragen dabei nicht nur im Sinne einer Reziprozität und Solidarität zu einem gewissen Ausgleich der Beziehungsasymmetrie bei, sondern erlauben in vielen Fällen auch einen guten Rückzug aus dem Feld bzw. gegebenenfalls die Transformation der Forschungsbeziehung in eine vom Forschungskontext losgelöste Bekanntschaft.

Sich einlassen, Geben und Nehmen, Flexibilität und Sensibilität sind für Forschung im Kontext von Verrechtlichung und Bürokratisierung sowie Unsicherheit und Exklusion ebenso notwendig, wie sich klar zu positionieren, den Forschungskontext immer wieder in den Vordergrund zu rücken und, wo nötig, Distanz zu schaffen. Erst indem man dem Kontext angepasst „mit Methoden und Verfahren ungefähr so umgeht wie ein Heimwerker mit seiner Ausrüstung, die nie genau auf das Problem zugeschnitten ist, das er konkret bewältigen muss" (Hitzler und Honer 1986, S. 23), können Daten generiert und der nächsten Herausforderung zugeführt werden: der Auswertung.

\section{Auswertung: Mehrsprachigkeit und Interkulturalität als Problem?}

Das Erkenntnisinteresse, intersubjektive Bedeutungen der Asylwirklichkeit zu erschließen, erfordert - wie viele andere Forschungsfragen - eine Auswertung, die nicht nur den Feld- und Kontextbedingungen gerecht wird, sondern über das Erschließen von manifesten Inhalten hinausgeht und auch Bedeutungen erfassen kann, die hinter explizit artikulierten Meinungen, Argumentationen oder Narrativen liegen.

Methodisch heißt dies, latente Bedeutungen und Hintergrundstrukturen zu rekonstruieren; ein Vorgehen, das z. B. die hermeneutische Wissenssoziologie für sich reklamiert (vgl. z. B. Schröer 1994; Soeffner und Hitzler 1994; Hitzler und Honer 1997; Soeffner 1989; Reichertz 2008, S. 519 ff.). Um latente Inhalte zu erschließen, geht der Weg meistens über die detaillierte Analyse von Sprechakten, die Verwendung von Begrifflichkeiten, Pausen, Betonungen etc. Im Fokus der Analyse stehen auch scheinbare „Belanglosigkeiten“, da gerade „die Art, wie jemand spricht, und nicht so sehr, was jemand inhaltlich sagt [wegweisend ist].“ (Froschauer und Lueger 2003, S. 102). Darüber hinausgehend sind auch kulturelle Bedeutungen wesentlich: So muss die Interpretin/der Interpret, ,eruieren, was die infrage stehenden Objektivationen im Hinblick auf kulturell bereit stehende ,Optionen ' bedeuten bzw. bedeuten können“ (Honer 1993, S. 109). Daher muss man

den sozio-kulturellen Zusammenhang, auf den der sich (Ent-)Äußernde bezieht, erkennen können und hinreichend - was immer das heißt - kennen, und man muß die Differenzen zwischen diesem und seinem eigenen kulturellen Kontext reflektieren und bei seinen Deutungen mitberücksichtigen (ebd.).

Die gegenständliche Studie war somit mit mehreren Schwierigkeiten konfrontiert, v. a. da die Interviewtranskripte von zweifacher Fremdheit geprägt waren: Erstens wurde keines der Interviews in der sogenannten ,Muttersprache“ der RespondentInnen geführt, sondern auf Deutsch bzw. bestenfalls auf Englisch oder Französisch. 
Zweitens unterschied sich „der kulturelle Hintergrund“ der RespondentInnen von „meinem kulturellen Hintergrund“ und von dem anderer AsylwerberInnen: eine Vielzahl an kulturellen Kontexten, die zu reflektieren und zu kennen sind.

Forschung im fremdsprachlichen und interkulturellen Kontext ist von ,unterschiedlichen Bedeutungsräumen der scheinbar gleichen Begriffe und [...] daraus resultierenden Unschärfen“ (Beck-Gernsheim 2003, S. 79) geprägt. Dabei stellt sich die Frage nach den Grenzen des wechselseitigen Verstehens, danach, inwiefern gemeinsame Bedeutungshorizonte überhaupt vorliegen bzw. ForscherIn und RespondentIn im (zusätzlich von Machtasymmetrien geprägten) Kontext überhaupt über „gemeinsame Symbolwelten“ (Tuider 2009) verfügen können. Offen ist, inwieweit in einem derartigen Kontext überhaupt Verstehen möglich ist, ohne dass derartige Versuche lediglich zu Missverständnissen und Fehlinterpretationen führen.

\section{Analyse Kultureller Positionierungen: Wessen und welche Sprache und Kultur?}

Die Lösungen, die die Literatur zu fremdsprachiger bzw. interkultureller qualitativer Forschung bietet, basieren meist auf der Einbeziehung von DolmetscherInnen, ÜbersetzerInnen oder AssistentInnen an unterschiedlichen Stellen des Forschungsprozesses. Inhetveen wurde in ihrer Forschung mit Flüchtlingen in Sambia von einem lokalen Forschungsassistenten unterstützt (Inhetveen 2012), in anderen Fällen werden Übersetzungen kommentiert (vgl. Tuider 2009, S. 189 f.), hoch qualifizierte Co-InterpretInnen bzw. „Kulturdolmetscher“ (Schröer 1998) herangezogen oder auf „muttersprachliche“ bzw. gemischtsprachige (Auswertungs-)Teams zurückgegriffen (vgl. z. B. Tuider 2009; Hangartner 2012). Empfehlungen zur Forschung ,in und mit fremden Sprachen“ (Kruse et al. 2012a) beziehen sich auf die verstärkte Einbeziehung von (finanziellen, sozialen und zeitlichen) Ressourcen, Forschung in der Sprache des Erhebungslandes und legen nahe, den Übersetzungszeitpunkt so spät wie möglich, d. h. bevorzugt bei der Auswertung anzusetzen (vgl. Kruse et al. 2012b, S. 52 ff.).

All diesen Lösungen ist gemein, dass spätestens bei der Interpretation der Interviews eine sprach- und/oder kulturkompetente Person unabdingbar wird. Abseits notwendiger Ressourcen für Co-ForscherInnen oder kulturkompetente InterpretInnen stellt sich insbesondere die Frage, wer diese Anforderungen im konkreten Forschungsfeld erfüllen kann: Welche Sprache(n) sollte eine Assistentin sprechen, welcher Kultur müsste der Co-Interpret angehören? Über welches Ausmaß an explizierbarem sprachlichem bzw. kulturellem Wissen müsste die Person verfügen? Brauche ich für alle meine InterviewpartnerInnen ein „Herkunfts-Pendant“ - d. h. für AsylwerberInnen aus Tschetschenien eine tschetschenische Assistentin, für somalische Befragte einen somalischen Co-Interpreten? Muss die Person (ehemalige/r) AsylwerberIn sein? Langansässige/r MigrantIn? ExpertIn des österreichischen Asylsystems? Inwiefern ist es notwendig, dass ein Interview mit einer muslimischen Frau aus Dagestan mit Unterstützung einer weiblichen, muslimischen Co-Interpretin aus Dagestan ausgewertet wird? Wer ist wirklich ExpertIn und potenzielle AssistentIn? Kurz: Um welche Sprache und welche Kultur geht es eigentlich? 
Wie in anderen Forschungskontexten an der Schnittstelle mehrerer Kulturen ist auch hier die Analyse kultureller Positionierungen ein erster notwendiger Schritt. Es gilt, den Blick zu sensibilisieren, um zu erkennen, welche Kultur(en) für ein Verstehen möglicherweise relevant ist (sind). Wenn beispielsweise Sharina in ihren Erzählungen von sich als Algerierin, Muslimin, Asylwerberin, Frau und Angehörige des Bildungsbürgertums spricht, wird schnell klar, dass es sich auf keinen Fall um eine Kultur (oder eine Sprache) handeln kann. Vielmehr zeigt sich, dass die vorhandenen Daten als an der Schnittstelle mehrerer - sowohl fremder als auch wohlbekannter - Sprachen und Kulturen produziert zu verstehen sind.

Für das Erschließen von Sinnstrukturen und Wahrnehmungsräumen reicht es demnach nicht, der Prämisse zu folgen, wonach ,der Forscher ein Vorwissen von der Besonderheit der Kulturräume haben [muss], die er behandelt. Ansonsten bleibt er hinterrücks in seinen eigenen Wahrnehmungsräumen befangen“ (Beck-Gernsheim 2003, S. 81). Vielmehr sind Hinweise auf ein Nebeneinander unterschiedlicher kultureller Bezugspunkte zu erkennen und es ist ein Aufbrechen des essentialisierten „kulturell Anderen“ notwendig, wie auch die Interviewten selbst nahelegen, wenn z. B. Sharina mehrfach auf ein ,wir Frauen“ Bezug nahm und Veronika unsere gemeinsame akademische Arbeitskultur hervorhob.

Statt Kultur zu essentialisieren, indem v. a. auf ethnische, nationale, religiöse oder sprachliche Merkmale Bezug genommen wird, ist im Sinne der Analyse kultureller Positionierungen der Blick auf (Sub-)Kulturen notwendig. Kulturelle Verortungen und somit der Bezug auf unterschiedliche Bedeutungssysteme, die mit entsprechenden sozialen Praktiken verbunden sind (vgl. Silbey 2001, S. 8624), sind dabei wandelbar und im Rahmen der Erzählung dynamisch. Wesentlich ist es, sich in der Auswertung dieser gemeinsamen und unterschiedlichen Bezugssysteme bewusst zu werden, um Grenzen des Verstehens zu erkennen bzw. Wege zu finden, Besonderheiten relevanter Kulturräume zu erschließen und diese für ein Verstehen der Asylwirklichkeit nutzbar zu machen.

\section{Fokus: Asylkultur}

Wenn die Frage gestellt wird, welche der Kulturen für die Forschung höchste Relevanz hat, rückt die „Asylkultur“ als solche ins Zentrum: Um zu erschließen, welcher (typische) Sinn Asyl zugeschrieben wird bzw. welche Konzeptionen ,des Flüchtlings“ bzw. „,des Asylwerbers“ in der österreichischen Asylwirklichkeit vorherrschen, ist es wesentlich, eben diese Wirklichkeit als Kulturraum zu verstehen und zu analysieren. Der Blick auf die Besonderheiten dieses Kulturraums wird notwendig, um weder in eigenen Wahrnehmungsräumen gefangen zu sein, noch sich in kulturellen Stereotypen der „MigrantInnen“, „Fremden“ oder ,Asylwerber“ zu verlieren.

Welchen Erkenntnisgewinn der Fokus auf die Asylkultur bringt, wird auch dann deutlich, wenn das Asylverfahren als Prozess der Akkulturation (Noll 2006, S. 497) verstanden wird. Noll argumentiert, dass es im Rahmen der Verfahren einerseits notwendig ist darzustellen, dass man einer Gruppe angehört, die aus Gründen verfolgt wird, die einer ,,anderen“ Kultur zuzurechnen sind; andererseits muss die Person 
aber eine „wahre Identität“ glaubhaft machen, die die kulturellen Werte des Aufnahmestaates vertritt (ebd., S. 496). Wenn nun - um Nolls Beispiel aufzugreifen ein Asylwerber seine Zugehörigkeit zur Subkultur der verfolgten Homosexuellen im Iran herausstreicht und sich gleichzeitig glaubhaft so positioniert, dass er die in Europa (zumindest rhetorisch) gepriesene Kultur der Menschenrechte und somit den Wert der Nicht-Diskriminierung aufgrund sexueller Orientierung in seiner Person verinnerlicht, hat er gute Chancen auf einen Flüchtlingsstatus.

Einer ähnlichen Logik folgend geben die in den Interviews artikulierten (und gegebenenfalls kommentierten) kulturellen Zugehörigkeiten Aufschluss darüber, wie „der Flüchtling“ oder „der Asylwerber" konstruiert wird und welche Bedeutungen kultureller Zugehörigkeit im Asylkontext vorherrschen. Ob und wie auf die Bitte, die Zeit als Asylwerber zu beschreiben, die Identität der ,westlichen Frau“"16, des „,verfolgten Opfers“, der Minderheit der Asharaf oder die des Lehrers oder der alleinerziehenden Mutter herausgestrichen werden, spielt für das Verständnis von dem, was ein ,echter Flüchtling“ sein darf, eine wesentliche Rolle. Wenn Anaida im Interview erklärt, dass sie gelernt hat, man dürfe als Asylwerberin weder gut angezogen sein, noch solle man Nagellack oder Parfum tragen, da jemand, der „eh super aus[schaut]“ (Anaida) keine asylrelevanten Probleme haben könne, wird ebenso auf die Symbolik und die Praktiken einer Asylkultur verwiesen.

Wenn Kultur als „'tool kit' of symbols, stories, rituals, and world-views, which people may use in varying configurations to solve different kinds of problems" (Swidler 1986, S. 273) verstanden wird, ist somit gerade das Erschließen dieses Toolkits über die Analyse relevant, um die Frage zu beantworten, welche Handlungsmöglichkeiten im Asylkontext genutzt werden, um mit den strukturellen Bedingungen der Asylwirklichkeit umzugehen.

\section{Perspektive Mehrsprachigkeit: Ils disent qu'il y a Freiheit ...}

Einer ähnlichen Logik folgend ist der Blick auch auf sprachliche Positionierungen zu werfen, wobei sich hier der Zugang der Mehrsprachigkeitsforschung als hilfreich erweist. Während die Literatur zur qualitativen Forschung im sogenannten fremdsprachigen Kontext meist (implizit) davon ausgeht, dass eine Person eine Mutter-, Erst- bzw. Hauptsprache spricht, verlässt die Mehrsprachigkeitsforschung diesen Zugang: Nicht die Sprache wird als Ausgangskategorie verstanden, sondern der Fokus liegt auf den SprecherInnen, denen, je nach Situationskontext, ein Repertoire an sprachlichen bzw. kommunikativen Möglichkeiten zur Verfügung steht (vgl. Slezak 2010, S. 10, 20). Dieses sogenannte Sprachrepertoire entwickelt sich u.a. über die Partizipation an verschiedenen sozialen Räumen, Sprachregimen, biografischen Momenten und über gesellschaftspolitische Faktoren. Das Sprachrepertoire von AsylwerberInnen ist somit nicht vorrangig auf deren nationales Sprachregime zurückzuführen, sondern in Zusammenhang mit der individuellen Biographie, die sich im Sprachrepertoire spiegelt, zu verstehen (vgl. Blommaert 2009, S. 423 f.).

16 Gerade für Frauen aus Afghanistan ist eine westliche Orientierung als Grund für einen Schutzstatus relevant. 
Als Personen, die per se „denationalized or transnationalized“ (ebd., S. 425) sind, verfügen AsylwerberInnen über translokale Biographien, die mit komplexen, polyglotten Sprachrepertoires einhergehen (vgl. Slezak 2010; Blommaert 2009; Busch 2010). Selbstverständlich prägen dabei auch das Asylsystem und die Lebenswelt als AsylwerberIn, d. h. die angesprochene „Asylkultur“, dieses Repertoire.

Aus diesem Zugang wird z. B: auch Deutsch - als Sprache des Asylverfahrens und offizielle Sprache des Aufnahmelands - zum Teil des Sprachrepertoires und somit $\mathrm{zu}$ einer Sprache der interviewten Person. ${ }^{17}$ Marika thematisiert dies ungefragt und explizit:

ich [...] merke, beim Fernsehen, wie ich nicht mehr übersetze von Georgisch auf Deutsch [...], manchmal, wenn ich den Einkaufszettel schreibe, schreib' ich Deutsch, mein Mann fragt mich dann, manche Wörter wie ,Sehenswürdigkeiten", die man nicht viel verwendet, die fallen mir dann auf Georgisch gar nicht ein. (Gesprächsprotokoll, 9.9.2010)

Entsprechend können RespondentInnen aktiv aus dem ihnen zur Verfügung stehenden (und durch die Sprachkenntnisse der Forscherin mitstrukturierten) Pool an heterogenen Ausdrucks- und Stilmitteln schöpfen, wie sich an der Sprechweise von Sharina ${ }^{18}$ zeigt, was folgender Zusammenschnitt illustriert:

Fekter, c'est wahnsinnig. [...] parce qu'ils [Anm.: die Ost-EuropäerInnen] sont un peu europäisch. [...] Je n'ai pas le droit de m'exprimer, je porte le Kopftuch. [...] tous les Ausländer doivent l'enlever; ils disent qu'il y a Freiheit, mais ce n'est pas le Freiheit. [...] Quand ma fille était née ils ont mis Staatsbürgerschaft Algerien. (Transkriptauszüge, 15.9.2010 und 9.11.2010)

Über den Zugang der Mehrsprachigkeitsforschung kommt es so zu einer Perspektivenverschiebung: Das Problem sind weniger Interviews in ,gebrochenem Deutsch“ oder ,kolonialem Französisch“, sondern vielmehr die Bedeutungen unterschiedlicher Begrifflichkeiten mit Hinblick auf das Erkenntnisinteresse, die Asylwirklichkeit zu verstehen, zum Teil der Analyse zu machen. ${ }^{19}$

\section{Fokus: Asylsprache}

Wenn, wie dargestellt, die „Asylkultur“ ins Zentrum rückt, so interessiert auch deren eigenes Sprachrepertoire - die „Asylsprache“. Diese ist, wie folgende Analyse zeigt, durch Überschneidungen und Aushandlungsprozesse geprägt:

\footnotetext{
17 Parallel gilt Ähnliches z. B. für Interviews auf Englisch oder Französisch, wenn z. B. Swahili oder Igbo die Sprachen wären, die gemeinhin als „Mutter- oder Erstsprache“ klassifiziert werden würden.

18 Sharina selbst gibt an, dass sie Arabisch, Französisch, Deutsch und etwas Englisch spreche; sie weiß, dass ich Deutsch, Englisch und Französisch verstehe und damit ihre Ausdrucksmöglichkeiten einschränke.

19 Dennoch bleiben die eingeschränkten Sprachkompetenzen von mir als Forscherin auf Ebene der Datenerhebung ein wesentliches Problem, da dadurch das Sample entsprechend eingeschränkt wird und Personen, die weder Deutsch noch Englisch oder Französisch zu ihrem Sprachrepertoire zählen, nicht befragt werden können.
} 
Bereits im Asylverfahren spielen Aspekte der Mehrsprachigkeit eine Rolle. Auch wenn sich in der deutschen Verfahrenssprache die monoglotte Ideologie im Asylkontext (vgl. Blommaert 2009 mit Bezug auf Silverstein) zeigt, braucht erfolgreiche Kommunikation im Verfahrenskontext zusätzliche Sprach(varietät)en, aus denen und in die gedolmetscht wird. Die AsylwerberInnen haben keine freie Wahl, was die Dolmetschsprache betrifft, sondern werden durch praktische Bedingungen, wie die Verfügbarkeit von (Laien-)DolmetscherInnen, ein begrenztes Zeitbudget und fehlende Ausbildungen in manchen Sprachen eingeschränkt (vgl. Slezak 2010, S. 47). Meist wird über eine kurze Befragung in einer Lingua Franca beurteilt, ob die Person ,fähig ist, in der gewählten Sprache verfahrensrechtliche Situationen zu beurteilen, und somit verfahrensrechtliche Ausdrücke [...] verstehen kann“ (vgl. Matti und Rienzner 2013, S. 3). Zurückgegriffen wird gegebenenfalls auf eine Sprache, ,deren Kenntnis vernünftigerweise vorausgesetzt werden kann und in der er [der Asylwerber] sich verständigen kann“ (EU-Verfahrensrichtlinie zit. in ebd., S. 4). Eine besondere Rolle spielen hier Amtssprachen, wie Arabisch, Russisch, Englisch oder Französisch; letztere insbesondere für AsylwerberInnen aus afrikanischen Ländern (vgl. Slezak 2010, S. 45 ff.; Matti und Rienzner 2013, S. 4). Dominante Amtssprachen, Deutsch als Verfahrenssprache plus eine weitere gedolmetschte Sprache und zusätzlich unterschiedliche Sprachvarietäten (z. B. Dialekte) der Beteiligten prägen das Verfahren. Die Vermischung von Sprachen scheint in rechtlichen Verfahren keine Seltenheit, denn in einem ,Mischmasch [...] geht dann die Verständigung noch eher“ (Zitat aus einem Interview in Slezak 2010, S. 47). Ähnliche Praktiken sind, u. a. aufgrund fehlender Ressourcen, auch in der Rechts- bzw. Sozialberatung, bei AnwältInnen oder im Rahmen der Betreuung in Flüchtlingshäusern zu beobachten.

Die herausragende Rolle und Präsenz der deutschen Sprache betrifft nicht nur das Verfahren: Die Verwendung deutscher Begrifflichkeiten in Zusammenhang mit dem Asylrecht oder der institutionellen Landschaft, wie z. B. Innenministerium, Schubhaft, ,Lagerkarte“, subsidiärer Schutz, Bundesasyl(amt), „Asylant“" oder Deutschkurs ist weit verbreitet. Im Alltag werden Deutschkenntnisse angesichts der Integrationsanforderungen nicht nur zu einer mächtigen Forderung, sondern auch zu einem wesentlichen kulturellen Kapital und ermöglichen Handlungsfähigkeit. Die Relevanz von Deutschkenntnissen zeigt sich bei Sharina einerseits durch ihren lückenlosen Sprachwechsel und andererseits durch die Thematisierung des Zusammenhangs zwischen Sprache, Integration und Anerkennung im Alltag:

[...] ils vont me dire, donnez-nous les, les certificats que vous avez fait, et faites l'interview en allemand, on ne veut pas une autre langue. Peut-être, hm, il faut que je parle là en allemand. Si je parle en allemand, et je leur donne le certificat, par example B1, zwei, et B2, ça c'est leur demande, integriert! Kann schon Deutsch sprechen, und sie hat schon ein Zertifikat. Das hat schon die Frau Strache, äh, Fekter gesagt, integriert und mit Zertifikat bis 2B geht schon. Ok? Ich mache was sie sagt!

[...] je voulais faire, äh, à ma fille un Sparbuch, et j'ai parlé avec quelqu'un, il m'a dit, combien d'années vous êtes ici? Woher kommen Sie, ich hab gesagt, aus Algerien. Wie lang sind Sie da, ich hab gesagt, äh, es war schon neun 
Jahre diese Zeit, schon, hat gesagt, aber mein Gott, Sie reden gut Deutsch! (Transkript, 9.11.2010)

Wenn also in der etablierten Methodenliteratur „Spracherwerb als Voraussetzung für erfolgreiche Feldforschung“" postuliert (Senft 2012, S. 123 ff.) und empfohlen wird, ,die Sprache des Erhebungslandes so gut wie möglich zu beherrschen“ (Kruse et al. 2012b, S. 53) bzw. ein „Vorwissen von der Besonderheit der Kulturräume“ (Beck-Gernsheim 2003, S. 81) für eine entsprechende Interpretation vorausgesetzt wird, stehen also - angesichts des Erkenntnisinteresses, die Asylwirklichkeit zu verstehen - insbesondere die Sprache und Kultur derselben im Zentrum.

\section{Forschungspraktische Auswertungszugänge}

Mit Bezug auf die Analyse kultureller Positionierungen und Prämissen der Mehrsprachigkeitsforschung ergeben sich auf auswertungspraktischer Ebene mehrere Zugänge, um ein Verstehen (bis auf weiteres) zu ermöglichen.

\subsection{Lesarten generieren durch existentielles Exponieren}

Um entsprechende Bedeutungen der Asylkultur zu erschließen, steht - wie in allen Zugängen, die auf latente Bedeutungen fokussieren - die Generierung möglichst unterschiedlicher Lesarten im Zentrum. Abseits gängiger Zugänge, wie z. B. der Interpretation in gemischten Teams, besteht eine Möglichkeit darin, sich der Asylkultur existenziell zu exponieren, mit ihrer Sprache und ihren Symbolen vertraut zu werden, um so auf neue Lesarten zu stoßen. D. h., sich dem Asylkontext reflektiert auszusetzen, sich mit möglichst unterschiedlichen Objektivationen der Asylwirklichkeit zu beschäftigen: Beobachtungen im Flüchtlingshaus, bei Beratungsstellen, Begleitungen zu Behörden, Teilnahme an Kundgebungen, Festen und öffentlichen Veranstaltungen, Lektüre von Zeitungsartikeln, Romanen oder Comics, Diskussionen über das Asylwesen in diversen Kontexten, Spaziergänge und Transect Walks zu relevanten Einrichtungen...

Anders als bei (teilnehmender) Beobachtung für die Datenerhebung steht hier der Aspekt der Auswertung im Zentrum: Im Sinne eines zyklischen Forschungsprozesses geht es darum, sich Irritationen auszusetzen, im Laufe der Forschung entstandene Hypothesen in Frage zu stellen, Widersprüche herzustellen oder aufzulösen, Bilder zu Erzählungen zu finden, Begriffen zu folgen, angesprochene Institutionen greifbar, erlebbar und fühlbar zu machen, das Vokabular der Asylkultur zu erlernen, ein Gefühl für Sprechweisen, Konflikte und Widersprüche zu entwickeln. Ein derartiges Sich-Einlassen ermöglicht somit über die Kontextualisierung und DeKontextualisierung ${ }^{20}$ von in den Interviews Angesprochenem neue Lesarten. Der Dialog mit „,dem Anderen“ (hier: der Asylkultur) und die Reflexion dieses Dialogs schaffen nicht nur Wissen, sondern ermöglichen es, ,harmonisierende Hypothesen

20 Nützlich sind hier auch Techniken, wie sie Strauss/Corbin im Rahmen der Grounded Theory vorschlagen, wie z. B. „Flip-Flop Technique“ oder „Far-Out Comparison“ (vgl. Strauss und Corbin 1990, S. 84 ff.). 
über den Relevanz- und Deutungsrahmen der fremden Kultur zu entwerfen und sie auf diese Weise bis auf weiteres zu verstehen“ (Schröer 1998, S. 162).

\subsection{Begriffssuche und Sprachlosigkeit als Chance}

Ein weiterer Weg zum Verstehen ist die Nutzbarmachung von kontextualisierenden Beschreibungen, sogenannten ,home narratives“ (Blommaert 2001, S. 428). Antizipierte kulturelle Unterschiede zwischen RespondentIn und InterviewerIn führen zu einem gewissen Erklärungszwang (vgl. Riemann 2003, Abs. 7); das Bemühen um eine gemeinsame Sprache bzw. ein gemeinsames Verständnis führt dazu, dass (kulturelle) Selbstverständlichkeiten nicht mehr als solche erscheinen und explizit thematisiert werden müssen (vgl. Kruse und Schmieder 2012, S. 254). Eine derartige „Ent-Selbstverständlichung“ (ebd., S. 271) wird häufig von den Befragten selbst eingebracht, in dem das Nicht-Verstehen der Interviewerin vorweggenommen und Dargelegtes expliziert wird: So erklärt z. B. Marika die politischen Realitäten in Georgien, damit ich ihre Verwunderung verstehe, dass der österreichische Präsident keinen relevanten Einfluss auf die Asylrealität hat:

Was Asyl angeht - es entscheidet alles nur Innenministerium [...] Herr Fischer kann nichts machen [...] bei uns ist nicht so, bei uns alles geht zum Präsident, er ist Oberchef für diese Staat und er ist verantwortlich und bei uns egal - vom Parlament entscheiden das ist so, und wir haben so so viele sind gegen und so viele sind für diese Gesetz und dann der entscheidet ja oder nein [...] und hier ist nicht so. Also im Asylgesetz. Herr, Frau Fekter ist Präsidentin. (Transkript, 23.9.2010)

Auf ähnliche Weise erklärt Andrej, warum er Österreich dankbar ist und wofür die Metapher des „Spazierengehens“ steht:

Für Österreich kann ich sagen nur vielen Dank, weil da ist sehr gut [...]. Meine Kinder ist kein Hunger, gibt es was anziehen, Kleidung [...] und das alles Österreich hat mir gegeben; in meinem Land auch nicht so keine Essen oder keine Anziehen, nicht das ist Problem, war, in meinem Land war Problem Sicherheit; in Österreich das ist ein Sicherheiten Land, und das ist sehr wichtig für mich [...] ich kann spazieren mit meinen Kindern wo ich will [...] das ist wichtig für mich. Bei uns nicht so Sicherheit. (Transkript, 13.9.2010)

Gerade wenn beide GesprächspartnerInnen davon ausgehen, dass sprachliche Lücken vorhanden sind, sinkt auch die Schwelle, diese explizit zu thematisieren, nachzufragen oder gemeinsam deren Bedeutung zu erschließen. Dialoghaft findet dann schon während des Gesprächs eine Art partizipative Interpretation statt, indem über Bedeutungen verhandelt wird (vgl. Kruse et al. 2012c, S. 17). Über die Methode der dreiphasigen Interviews kann der methodische Vorteil der besonderen Sprachsituation (vgl. ebd., S. 40 f.) insofern genutzt werden, als dass erste Lesarten in einer zweiten Phase direkt geprüft oder erweitert werden können. Das Nachfragen zu Unklarheiten vorangegangener Phasen führt zu neuen Lesarten: Wenn Erzählungen als „creative constructions or interpretations of the past, generated in specific contexts of the present" (Eastmond 2007, S. 250) verstanden werden, kann nicht davon ausge- 
gangen werden, dass die zeitlich versetzte Erklärung unbedingt mit dem subjektiven Sinn, der mit der ursprünglichen Äußerung verbunden war, übereinstimmt. Vielmehr ist es der/die RespondentIn, die mit ihrem kulturellen, sprachlichen und lebensweltlichen Wissen nachträglich das Gesagte in einem ersten Schritt interpretiert, d.h. eine zusätzliche Lesart für das Verstehen bietet.

\subsection{Die Daten ernst nehmen}

Das Gegenüber und seine Äußerungen ernst zu nehmen, gilt als Prämisse während der Datenerhebung und Analyse. Wenn nämlich nicht davon ausgegangen wird, „dass eine Interviewperson das sagt, was sie sagen will [...], wird Verstehen respektive Analyse zu einem autoritativen Ratespiel“" (Kruse und Schmieder 2012, S. 266). In dem Sinne sind sprachliche Besonderheiten und/oder (kulturelle) Fremdheiten und Irritationen nicht einfach als Lücken auf dem Weg zum Verstehen zu sehen, sondern als ein Aspekt der Übermittlung von Inhalten. Gerade der Blick auf prägnante Stellen, die auf den ersten Blick wenig Sinn ergeben, überraschend klar sind, sprachlich holprig oder widersprüchlich erscheinen oder an denen ein sprachlicher Wechsel stattfindet, führt zu neuen Erkenntnissen, wie folgende Beispiele illustrieren:

So verwendet z. B. Andrej im Interview die Phrase ,ich bin Asyl“". Eine lückenorientierte Interpretation korrigiert „Asyl“ auf ,Asylwerber" und versteht den Ausdruck als Selbstpositionierung. Wenn aber die sprachlich prägnante Stelle stutzig macht und - mit Rückgriff auf andere Stellen im Transkript - einer genaueren Analyse unterzogen wird, eröffnet sich eine Lesart, die das Identitäre des Asylwerber-Seins betont, zeigt, wie notwendig es ist, bis zum Verfahrensabschluss ganz und gar ,Asyl zu sein"; d. h. alle mit der Institution Asyl verbundenen Charakteristika in die eigene Identität und die nach außen gerichtete Selbstdarstellung zu übernehmen.

Die Analyse von Widersprüchen wurde bei Belisha relevant: Während der ersten Treffen verwendet sie den Begriff "Integration“ aktiv und, wie ich glaube, in vollem Wissen um dessen Bedeutung. Gegen Ende unserer Forschungsbeziehung lädt sie mich zum Fastenbrechen ein. Während des Essens fragt sie mich plötzlich: „Andrea, was ist denn das eigentlich, diese Integration?“ Überrascht, dass ihr der Begriff trotz der mehrfach korrekten Verwendung fremd zu sein scheint, schaue ich mir in den Interviews die Stellen an, in denen der Begriff Anwendung findet: Fast überall wurde der Begriff in Zusammenhang mit Erzählungen von Briefen von Bekannten, die Belisha's „Integration“ bestätigen, von Deutschzertifikaten, die sie der Behörde vorgelegt hat und von behördlichen Forderungen, Beweise für die Integration zu erbringen, in Verbindung gebracht. Integration bezeichnet somit eine Art der „Vertextlichung“ ihrer österreichischen Lebenswelt, ein Dokument, das sie ihrem Asylakt beisteuert, in der Hoffnung, so einen rechtlichen Status zu erlangen. Die Analyse des vermeintlich klaren Begriffs spiegelt die durch Bürokratisierung entstandene Begriffsleere wider, indem Integration auf Papier, Text und Aktenteile reduziert wird und mit tatsächlicher Teilhabe am österreichischen Leben nichts mehr gemein hat.

Als besonders fruchtbar erweist sich auch die Analyse des Sprachkontexts bzw. der Sprach- bzw. Verständigungskompetenz, wie das Gespräch mit Sediq zeigt. Der 
afghanische Asylwerber beginnt - ohne dass ich eine Frage stelle - zu erzählen. Ohne Unterbrechung spricht er in gutem und in weiten Teilen überraschend korrekten Deutsch ${ }^{21}$ über seine Heimat und beschreibt die Stationen seiner Flucht. Nachdem die Narration in Österreich angekommen ist, bittet er mich explizit um eine weitere Frage, woraufhin ich ihn bitte, mir von seinem Asylverfahren zu erzählen. Plötzlich stockt die Erzählung und die Verständigung wird sprachlich schwierig. In der Analyse wird klar, dass die deutsche Sprachkompetenz genau für die Erzählungen zur Verfügung steht, die im Asylverfahren eine Rolle spielen und auch als Legitimierung des ,echten Flüchtlings (als Opfer)“ dienen: eine gefährliche Reise am Landweg von Afghanistan nach Österreich sowie menschenunwürdige und von Gewalt geprägte Bedingungen im Heimatland. Gerade der Blick auf die sprachlichen Bruchstellen ermöglicht so Erkenntnisse über Bedeutungen, die mit „dem legitimen Flüchtling“ in Verbindung stehen und deren Darstellung das Verfahren immer wieder einfordert.

Um die Asylkultur über Interviews mit fremdsprachlichen und interkulturellen Bezügen zu erschließen, sind es somit gerade sprachliche Irritationen, die für die Identifizierung relevanter Stellen und für verstehensorientierte Übersetzungen an der Schnittstelle diverser kultureller Positionierungen nutzbar gemacht werden können. Um zu verstehen, ist nicht nur De-Kontextualisierung sinnvoll, sondern v. a. auch Kontextualisierung notwendig. Bedeutungen bestimmter Stellen müssen über Ausführungen anderer Stellen des Interviews erschlossen werden und Informationen „von außen“ sind insbesondere für ein Verstehen der Asylkultur heranzuziehen, um „die Bedeutung im Kontext [zu] erhellen“ (Beck-Gernsheim 2003, S. 87).

Und auch für die Asylwirklichkeit, die auf den ersten Blick mehrfach von Fremdheit geprägt zu sein scheint, gilt, dass ,qualitative Sozialforschung eigentlich stets im Fremdsprachenkontext bzw. im interkulturellen Kontext stattfindet - und die Analyse von Sprache immer eine Navigation in fremden [und auch heimischen Anm. A. F.] Gewässern ist“" (Kruse und Schmieder 2012, S. 291).

\section{Conclusio}

Wenn die Asylwirklichkeit das Forschungsinteresse bestimmt, wird das Gegenüber in der Rolle bzw. Zuschreibung als Asylwerber adressiert. Dies impliziert methodische Zugänge, die nicht zuvorderst auf die Migrantin oder den Flüchtling als hilfs- und schutzbedürftige Person, sondern vielmehr am gesellschaftlichen bzw. institutionellen Umfeld auszurichten sind, das Asylwerber schafft und deren damit einhergehende Lebenswelt bedingt. Ein Blick, der vorrangig auf die strukturellen Rahmenbedingungen fokussiert, welche die Identitäten, Handlungsorientierungen und Ausdrucksmöglichkeiten von RespondentInnen prägen, hat andere methodische Konsequenzen als die Ausrichtung des Erhebungsinstruments an den Charakteristika des Individuums. Ein Erhebungsinstrument, das Bedingungen der Verrechtlichung und Bürokratisierung, Unsicherheit und Exklusion unberücksichtigt lässt, läuft Gefahr, Erzählmuster behördlicher Einvernahmen in den Daten zu reproduzieren und Unsicherheiten zu zementieren.

\footnotetext{
${ }^{21}$ Sediq ist (erst) seit sieben Monaten in Österreich.
} 
Im konkreten Fall sind die Erhebungsmethoden einem durch starke Machtasymmetrien geprägten Kontext anzupassen, in dem parallel zur Forschung behördliche „Interviews“" stattfinden, die über Bleiben (dürfen) oder Gehen (müssen), Recht oder Rechtlosigkeit entscheiden. Gefordert werden einerseits das bewusste Umdrehen der Merkmale behördlicher „Interviews“ und andererseits eine flexible und kreative Handhabe gängiger Ansätze, wie z. B. des dreiphasigen Intensivinterviews der lebensweltlichen Ethnographie. Notwendig ist eine Balance zwischen Nähe, Solidarität, existenziellem Sich-Einlassen und Rollenklarheit sowie professioneller Distanz. Anders als „reciprocal research“ im Sinne Pittaways et al. (2010, S. 238) erfordert reziproke Forschung im vorliegenden Kontext von der Forscherin mehr als eine gute, narrationsgenerierende Zuhörerin bzw. Interviewerin zu sein und zu gewährleisten, dass die Forschung den Beforschten zugutekommt. Reziprozität bedeutet darüber hinaus, sich einerseits aktiv als Person ins Interview einzubringen und zu positionieren und andererseits Raum zu schaffen, damit die InterviewpartnerInnen passende Gegenleistungen aktiv einfordern können.

Auf der Ebene der Auswertung steht das Erschließen von latenten Bedeutungen vor Herausforderungen: Gesellschaftliche Diskurse, in denen Flucht per se ein „Problem der Anderen“ ist, die „organisierte Desintegration“ (Täubig 2009) der Grundversorgung, die Bedeutung der Zugehörigkeit zur ,anderen Kultur“ für einen positiven Verfahrensabschluss sowie monoglotte Ideologien im Asylkontext (vgl. Blommaert 2009 mit Bezug auf Silverstein) führen nicht nur zu einer sozialen und geographischen Exklusion, sondern zeichnen auch ein Bild des ,,absolut Fremden“, dessen Bedeutungszuschreibungen kaum erschließbar erscheinen. Auch wenn aktuelle Forschungen im Asylkontext diese Diskurse aufbrechen und die Verhältnisse kritischer beleuchten, bleibt im Bereich der Migrations- und Flüchtlingsforschung auf methodischer Ebene dennoch die Suche nach „,kulturkompetenten“ und ,,muttersprachlichen" Co-ForscherInnen und InterpretInnen. Die u. a. durch Herkunft, Ethnizität oder Sprache bedingten Unterschiede sind jedoch nicht nur als Hindernisse am Weg zum Verstehen zu sehen, sondern insbesondere auch als Chance; gerade daraus resultierende Irritationen können nutzbar gemacht werden, um neue Lesarten für das Verständnis der eigentlich interessierenden Kultur - der Asylkultur - zu generieren. Sprachkontext und -irritationsanalysen sowie Fokussierungen auf kulturelle Positionierungen bieten eine Möglichkeit, den Blick nicht nur auf das Erzählte zu lenken, sondern auf das, was im Kontext erzählbar ist und was - in der Asylkultur und -sprache - erzählt werden kann. Entsprechende Einschränkungen sind in der Auswertung weniger als Folge eines Missverhältnisses zwischen InterviewerIn und RespondentIn, sondern vielmehr als strukturell bedingte Begrenzungen der Erzählungen und Re-Präsentationen zu denken. AsylwerberInnen sind entsprechend als RespondentInnen weniger als MigrantInnen mit einer anderen Kultur und Sprache interessant denn als RepräsentantInnen und v. a. als ExpertInnen dieser Asylkultur und deren Sprache. 
Open access funding provided by University of Vienna.

Open Access Dieser Artikel wird unter der Creative Commons Namensnennung 4.0 International Lizenz (http://creativecommons.org/licenses/by/4.0/deed.de) veröffentlicht, welche die Nutzung, Vervielfältigung, Bearbeitung, Verbreitung und Wiedergabe in jeglichem Medium und Format erlaubt, sofern Sie den/die ursprünglichen Autor(en) und die Quelle ordnungsgemäß nennen, einen Link zur Creative Commons Lizenz beifügen und angeben, ob Änderungen vorgenommen wurden.

\section{Literatur}

Asylkoordination Österreich. 2009. Politische Partizipation und Repräsentanz von Flüchtlingen und AsylwerberInnen in der EU. Forschungsbericht im Rahmen des Programms New orientations for Democracy in Europe > node 〈. Synthese der Fallstudien in Österreich, Frankreich und der Tschechischen Republik. http://www.asyl.at/projekte/node/synthese_casestudies.pdf. Zugegriffen: 14.05.2014.

Bauböck, Rainer. 2004. Migration und innere Sicherheit: Komplexe Zusammenhänge, paradoxe Effekte und politische Simplifizierungen. Österreichische Zeitschrift für Politikwissenschaft 33:49-66.

Beck-Gernsheim, Elisabeth. 2003. Interkulturelle Missverständnisse in der Migrationsforschung. Leviathan 31:72-91. doi:10.1007/s11578-003-0004-2.

Benhabib, Seyla. 2006. The rights of others. Aliens, residents, and citizens. Cambridge: Cambridge University Press.

Bigo, Didier. 2010. Sicherheit und Immigration: Zu einer Kritik der Gouvernementalität des Unbehagens. In Politik der Unentschiedenheit. Die internationale Politik und ihr Umgang mit Kriegsflüchtlingen, Hrsg. Margarete Misselwitz, 39-75. Bielefeld: transcript.

Block, Karen, Deborah Warr, Lisa Gibbs, und Elisha Riggs. 2012. Addressing ethical and methodological challenges in research with refugee-background young people: reflections from the field. Journal of Refugee Studies 26:69-87. doi:10.1093/jrs/fes002.

Blommaert, Jan. 2001. Investigating narrative inequality: African asylum seekers' stories in Belgium. Discourse \& Society 12:413-449. doi:10.1177/0957926501012004002.

Blommaert, Jan. 2009. Language, asylum, and the national order. Current Anthropology 50:415-441. doi: $10.1086 / 600131$.

BM.I. 2012. Asylstatistik 2012. http://www.bmi.gv.at/cms/BMI_Asylwesen/statistik/files/2013/ Asylstatistik_Jahr_2012.pdf. Zugegriffen: 14.05.2014.

Bourdieu, Pierre. 2010. Verstehen. In Das Elend der Welt. Gekürzte Studienausgabe, Hrsg. Pierre Bourdieu et al., 393-426. Konstanz: UVK. Erstauflage 1993.

Busch, Brigitta. 2010. ...und Ihre Sprache? Über die Schwierigkeiten, eine scheinbar einfache Frage zu beantworten. Stichproben. Wiener Zeitschrift für kritische Afrikastudien 10:9-33.

Conlon, Deirdre. 2013. Becoming legible and 'legitimized': subjectivation and governmentality among asylum seekers. In Migrant marginality: a transnational perspective, Hrsg. Jorge Capetillo-Ponce, Glen Jacobs, und Phil Kretsedemas, 186-204. New York London: Routledge.

Cuttitta, Paolo. 2010. Das europäische Grenzregime: Dynamiken und Wechselwirkungen. In Grenzregime. Diskurse, Praktiken, Institutionen in Europa, Hrsg. Sabine Hess, und Bernd Kasparek, 23-42. Berlin: Assoziation A.

Eastmond, Marita. 2007. Stories as lived experience: narratives in forced migration research. Journal of Refugee Studies 20:248-264. doi:10.1093/jrs/fem007.

ECRE - European Council on Refugees and Exiles. 2013. Not there yet: an NGO perspective on challenges to a fair and effective common European asylum system. http:/www.asylumineurope.org/sites/ default/files/shadow-reports/not_there_yet_02102013.pdf. Zugegriffen: 08.09.2016.

Ellis, Heidi B., Maryam Kia-Keating, Aden Yusuf Siraad, Alisa Lincoln, und Abdirahman Nur. 2007. Ethical research in refugee communities and the use of community participatory methods. Transcultural Psychiatry 44:459-489. doi:10.1177/1363461507081642.

Flick, Uwe. 2008. Triangulation in der qualitativen Forschung. In Qualitative Forschung. Ein Handbuch, Hrsg. Uwe Flick, Ernst von Kardorff, und Ines Steinke, 309-378. Reinbek bei Hamburg: Rowohlt TB.

Fritsche, Andrea. 2012. Zeit. Macht. Flüchtlinge. Und Flüchtlinge machen Zeit. Konzeptionen biographischer Zeiten im Asylkontext. SWS-Rundschau 52:362-388.

Fritsche, Andrea. 2013. Asylum seekers - Legal objects or legal subjects? Consequences of asylum law, room for manoeuvre and the necessity of agency - an asylum seekers' perspective. In The role of the 
$E U$ in UN human rights reform. Studienreihe des Ludwig Boltzmann Instituts für Menschenrechte, Bd. 29, Hrsg. Manfred Nowak, Tina Hofstätter, und Jane Hofbauer, 153-164. Wien Graz: NWV.

Fröhlich, Christian. 2012. Interviewforschung im russisch-sprachigen Raum - ein Balanceakt zwischen methodologischen und feldspezifischen Ansprüchen. In Qualitative Interviewforschung in und mit fremden Sprachen. Eine Einführung in Theorie und Praxis, Hrsg. Jan Kruse, Stephanie Bethmann, Debora Niermann, und Christian Schmieder, 186-202. Weinheim: Beltz Juventa.

Froschauer, Ulrike, und Manfred Lueger. 2003. Das qualitative Interview. Zur Praxis interpretativer Analyse sozialer Systeme. Wien: WUV.

Hangartner, Judith. 2012. Verstehen und „kulturelles Übersetzen“ in einer anthropologischen Feldforschung. In Qualitative Interviewforschung in und mit fremden Sprachen. Eine Einführung in Theorie und Praxis, Hrsg. Jan Kruse, Stephanie Bethmann, Debora Niermann, und Christian Schmieder, 136-150. Weinheim: Beltz Juventa.

Hitzler, Ronald, und Anne Honer. 1986. Zur Ethnographie kleiner Lebens-Welten. Theorie- und Methodenbasteln im Kontext des „Heimwerker“-Projekts. Forschungsbericht Nr. 2. Bamberg: Universität Bamberg.

Hitzler, Ronald, und Anne Honer. 1997. Sozialwissenschaftliche Hermeneutik. Eine Einführung. Opladen: Leske + Budrich.

Honer, Anne. 1993. Lebensweltliche Ethnographie. Ein explorativ-interpretativer Forschungsansatz am Beispiel von Heimwerker-Wissen. Wiesbaden: Deutscher Universitäts-Verlag.

Honer, Anne. 2008. Lebensweltanalyse in der Ethnographie. In Qualitative Forschung. Ein Handbuch, Hrsg. Uwe Flick, Ernst Kardoff, und Ines Steinke, 194-204. Reinbek bei Hamburg: Rowohlt.

Inhetveen, Katharina. 2012. Translation Challenges: Qualitative Interviewing in a Multi-Lingual Field. Qualitative Sociology Review 8:28-45.

König, Alexandra, und Sieglinde Rosenberger. 2010. Desintegration, Dezentralität, Disziplinierung: Grundversorgung im Bundesländervergleich. In Asylpolitik in Österreich. Unterbringung im Fokus, Hrsg. Sieglinde Rosenberger, 272-295. Wien: Facultas.

Kruse, Jan, und Christian Schmieder. 2012. In fremden Gewässern. Ein integratives Basisverfahren als sensibilisierendes Programm für rekonstruktive Analyseprozesse im Kontext fremder Sprachen. In Qualitative Interviewforschung in und mit fremden Sprachen. Eine Einführung in Theorie und Praxis, Hrsg. Jan Kruse, Stephanie Bethmann, Debora Niermann, und Christian Schmieder, 248-295. Weinheim: Beltz Juventa.

Kruse, Jan, Stephanie Bethmann, Debora Niermann, und Christian Schmieder. 2012a. Qualitative Interviewforschung in und mit fremden Sprachen. Eine Einführung in Theorie und Praxis. Weinheim: Beltz Juventa.

Kruse, Jan, Stephanie Bethmann, Debora Niermann, und Christian Schmieder. 2012b. In und mit fremden Sprachen forschen. Eine empirische Bestandsaufnahme zu Erfahrungs- und Handlungswissen von Forschenden. In Qualitative Interviewforschung in und mit fremden Sprachen. Eine Einführung in Theorie und Praxis, Hrsg. Jan Kruse, Stephanie Bethmann, Debora Niermann, und Christian Schmieder, 27-68. Weinheim: Beltz Juventa.

Kruse, Jan, Stephanie Bethmann, Debora Niermann, und Christian Schmieder. 2012c. Qualitative Interviewforschung im Kontext fremder Sprachen. Eine Einleitung. In Qualitative Interviewforschung in und mit fremden Sprachen. Eine Einführung in Theorie und Praxis, Hrsg. Jan Kruse, Stephanie Bethmann, Debora Niermann, und Christian Schmieder, 9-26. Weinheim: Beltz Juventa.

Mackenzie, Catriona, Christopher McDowell, und Eileen Pittaway. 2007. Beyond 'do no harm': the challenge of constructing ethical relationships in refugee research. Journal of Refugee Studies 20:299-319. doi:10.1093/jrs/fem008.

Matti, Emanuel, und Martina Rienzner. 2013. Dolmetschen und Übersetzen im Asylverfahren. migraLex 11:2-13.

Müller, Doreen. 2010. Flucht und Asyl in europäischen Migrationsregimen. Metamorphosen einer umkämpften Kategorie am Beispiel der EU, Deutschlands und Polens. Göttingen: Universitätsverlag.

Noll, Gregor. 2006. Asylum claims and the translation of culture into politics. Texas International Law Journal 41:491-501.

Pittaway, Eileen, Linda Bartolomei, und Richard Hugman. 2010. 'Stop Stealing Our Stories': The Ethics of Research with Vulnerable Groups. Journal of Human Rights Practice 2:230-251. doi:10.1093/ jhuman/huq004.

Reichertz, Jo. 2008. Objektive Hermeneutik und hermeneutische Wissenssoziologie. In Qualitative Forschung. Ein Handbuch, Hrsg. Uwe Flick, Ernst Kardoff, und Ines Steinke, 514-524. Reinbek bei Hamburg: Rowohlt. 
Riemann, Gerhard. 2003. A joint project against the backdrop of a research tradition: an introduction to „,doing biographical research“ [36 paragraphs]. Forum Qualitative Sozialforschung 4:Art. 18. http:// nbn-resolving.de/urn:nbn:de:0114-fqs0303185.

Rodgers, Graeme. 2004. 'hanging out' with forced migrants. Methodological and ethical challenges. Forced Migration Review 21:48-49.

Sales, Rosemary. 2002. The deserving and the undeserving? Refugees, asylum seekers and welfare in Britain. Critical Social Policy 22:456-478. doi:10.1177/026101830202200305.

Scheffer, Thomas. 2003. Kritik der Urteilskraft - Wie die Asylprüfung Unentscheidbares in Entscheidbares überführt. In Migration steuern und verwalten. Deutschland vom späten 19. Jahrhundert bis zur Gegenwart, Hrsg. Jochen Oltmer, 423-458. Göttingen: V \& R Unipress.

Schröer, Norbert. 1994. Interpretative Sozialforschung. Auf dem Wege zu einer hermeneutischen Wissenssoziologie. Opladen: Westdeutscher Verlag.

Schröer, Norbert. 1998. Kommunikationskonflikte zwischen deutschen Vernehmungsbeamten und türkischen Migranten. Soziale Probleme 9:154-181.

Schumacher, Sebastian, Johannes Peyrl, und Thomas Neugschwendtner. 2012. Fremdenrecht. Asyl. Ausländerbeschäftigung. Einbürgerung. Einwanderung. Verwaltungsverfahren. Wien: ÖGB.

Senft, Gunter. 2012. Das Erlernen von Fremdsprachen als Voraussetzung für erfolgreiche Feldforschung. In Qualitative Interviewforschung in und mit fremden Sprachen. Eine Einführung in Theorie und Praxis, Hrsg. Jan Kruse, Stephanie Bethmann, Debora Niermann, und Christian Schmieder, 121-135. Weinheim: Beltz Juventa.

Silbey, Susan S. 2001. Legal culture and legal consciousness. In International encyclopedia of social and behavioral sciences, Hrsg. Neil J. Smelser, und Paul B. Baltes, 8623-8629. Amsterdam: Elsevier.

Slezak, Gabriele. 2010. Mehrsprachig im einsprachigen Kontext: Einschätzung von Sprachkompetenz und Gestalten von Rahmenbedingungen für Kommunikation bei Gerichten und Behörden in Österreich. Stichproben. Wiener Zeitschrift für kritische Afrikastudien 10:35-61.

Soeffner, Hans-Georg. 1989. Der Alltag der Auslegung - Der Alltag der Auslegung. Zur wissenssoziologischen Konzeption einer sozialwissenschaftichen Hermeneutik. Frankfurt am Main: Suhrkamp.

Soeffner, Hans-Georg, und Ronald Hitzler. 1994. Hermeneutik als Haltung und Handlung. Über methodisch kontrolliertes Verstehen. In Interpretative Sozialforschung. Auf dem Wege zu einer hermeneutischen Wissenssoziologie, Hrsg. Norbert Schröer. Opladen: Westdeutscher Verlag.

Strauss, Anselm L., und Juliet M. Corbin. 1990. Basics of qualitative research. Grounded theory procedures and techniques. Newbury Park: SAGE.

Swidler, Ann. 1986. Culture in action: symbols and strategies. American Sociological Review 51:273-286.

Täubig, Vicki. 2009. Totale Institution Asyl. Empirische Befunde zu alltäglichen Lebensführungen in der organisierten Desintegration. Weinheim: Juventa.

Thielen, Marc. 2009. Freies Erzählen im totalen Raum? - Machtprozeduren des Asylverfahrens in ihrer Bedeutung für biografische Interviews mit Flüchtlingen. Forum Qualitative Sozialforschung 10:Art. 39. http://nbn-resolving.de/urn:nbn:de:0114-fqs0901393.

Tuider, Elisabeth. 2009. Transnationales Erzählen. Zum Umgang mit Über-Setzungen in der Biographieforschung. In Gender Mobil? Geschlecht und Migration in transnationalen Räumen, Hrsg. Helma Lutz, 174-192. Münster: Westfälisches Dampfboot.

UNHCR. 2013. Handbuch und Richtlinien über Verfahren und Kriterien zur Feststellung der Flüchtlingseigenschaft gemäß dem Abkommen von 1951 und dem Protokoll von 1967 über die Rechtsstellung der Flüchtlinge. http://www.unhcr.at/fileadmin/rechtsinfos/fluechtlingsrecht/1_international/1_ 1_voelkerrecht/1_1_2/FR_int_vr_handb-Handbuch.pdf. Zugegriffen: 08.09.2016.

Andrea Fritsche ist Doktoratsstudentin am Institut für Soziologie, Lehrbeauftragte an der Universität Wien, ehrenamtliche Rechts- und Sozialberaterin im Asylkontext; Forschungsinteressen: Asyl und Migration, Recht und Gesellschaft, qualitative Methode. 